\title{
A PRÁTICA E O CONHECIMENTO DA MULHER IDOSA PARA O ENVELHECIMENTO ATIVO
}

\section{THE PRACTICE AND KNOWLEDGE OF THE ELDERLY WOMAN FOR ACTIVE AGING}

\author{
Larissa Silva Santos ${ }^{1} *$ Monica Cavalcante ${ }^{2} *$ Tatiane Barbosa de Lira $^{3} *$ Natanael Nunes da $^{*}$ \\ Silva $^{4} *$ Armany Rickiel Lima Borges $5 *$ Pabline Kaiane Ferreira Jacobina $^{6} *$ Daiane $^{2}$ \\ Monique de Sá Martins ${ }^{7}$ * Francisca Cecília Viana Rocha ${ }^{8}$
}

\begin{abstract}
RESUMO
Objetivos: Analisar e descrever a prática e o conhecimento da mulher idosa sobre como manter um envelhecimento ativo e saudável e as dificuldades encontradas. Métodos: Trata-se de uma pesquisa descritiva, com abordagem qualitativa, realizada em um Centro de Convivência, de caráter público, na cidade de Teresina, Piauí. Os participantes do estudo foram 12 mulheres idosas que frequentem o Centro de Convivência. A análise dos dados foi realizada por meio da técnica de análise de conteúdo, cuja finalidade é analisar a partir das falas dos entrevistados. Resultados: Foi analisado o perfil sociodemográico das idosas e também abordou as categorias temáticas do estudo, de acordo com a similaridade semântica, que representam as descrições das falas das idosas entrevistadas que foram as práticas das idosas para um envelhecimento saudável e ativo e dificuldades das idosas para o envelhecimento ativo. Conclusão: $O$ envelhecimento ativo é encarado como um processo de otimização das oportunidades de saúde, participação e segurança.
\end{abstract}

Descritores: Idosa. Envelhecimento Ativo. Saúde da Mulher.

\begin{abstract}
Objectives: Analyze and describe the practice and knowledge of the elderly woman about how to maintain an active and healthy aging and the difficulties encountered. Methods: This is a descriptive research with a qualitative approach, performed at a Center for Coexistence of public character, in the city of Teresina, Piaui. The study participants were 12 elderly women who attend the Center for Coexistence. The data analysis was performed by means of the content analysis technique, whose purpose is to analyze the effect of the statements of the interviewees. Results: We analyzed the sociodemográico profile of elderly people and also addressed the thematic categories of the study, in accordance with the semantic similarity, which represent the descriptions of the statements of the elderly interviewees who were the practices of elderly people for a healthy and active aging and difficulties of elderly people for active aging. Conclusion: The active aging is seen as a process of optimizing opportunities for health, participation and security.
\end{abstract}

Keywords: Elderly. Active Aging. Women's Health.

\footnotetext{
${ }^{1}$ Enfermeira. Pós-graduanda em Urgência e Emergência pela IESM, Timon, Piauí. Brasil.

${ }^{2}$ Enfermeira. Especialista em Unidade de Terapia Intensiva, Uniredentor/AMIB, Teresina, Piauí. Brasil.

${ }^{3}$ Enfermeira. Residente em Cuidados Intensivos pelo Hospital Universitário da Universidade Federal do Piauí, Teresina. Piauí. Brasil

${ }^{4}$ Enfermeiro. Graduado em Enfermagem pela Universidade Estadual do Piauí - UESPI, Teresina, Piauí. Brasil.

${ }^{5}$ Enfermeiro. Pós-graduando em Enfermagem em Terapia Intensiva pela Associação Piauiense de Combate ao Câncer Alcenor Almeida - APCCAA - Hospital São Marcos. Teresina, Piauí. Brasil.

${ }^{6}$ Enfermeira. Graduada em Enfermagem pelo Centro Universitário UNINOVAFAPI. Teresina. Piauí. Brasil.

${ }^{7}$ Enfermeira. Pós-graduanda em Unidade de Terapia Intensa pelo Hospital São Marcos. Teresina, Piauí. Brasil.

8 Enfermeira. Mestre em Enfermagem. Docente do Departamento de Enfermagem do Centro Universitário UNINOVAFAPI.

Teresina. Piauí. Brasil.
} 


\section{INTRODUÇÃO}

O processo do envelhecimento pode ser classificado de dois modos: a senescência ou envelhecimento primário e a senilidade ou envelhecimento secundário. A senescência consiste nas mudanças causadas pela idade, independentes das doenças e das influências do ambiente. A senilidade consiste numa aceleração deste processo como resultado das doenças que surgem ocasionalmente, assim como, dos fatores ambientais ou doenças crônicas $^{(1)}$.

$\mathrm{O}$ envelhecimento ativo tem como objetivo "aumentar a expectativa de uma vida saudável e a qualidade de vida para todas as pessoas" que se encontram neste processo. É importante salientar que a Organização Mundial da Saúde (OMS) associa o termo "ativo" não somente à capacidade de estar fisicamente $\operatorname{ativo}^{(2)}$.

O envelhecimento saudável é definido por medidas subjetivas, tais como: satisfação de vida, afetos e disposição de espírito, e por medidas objetivas, nomeadamente, morbidade, independência e mortalidade. A partir de uma perspectiva biomédica, a definição mais proeminente descreve-o em termos de três critérios: baixo risco para doenças e deficiências relacionadas à doença, alta atividade mental e física e envolvimento ativo na vida cotidiana. $\mathrm{Na}$ perspectiva psicossocial, o bem-estar emocional é uma das dimensões mais valorizadas. Relaciona-se com o que vem a ser uma vida satisfatória e feliz e tem sido explicado por dois modelos (3).

$\mathrm{O}$ entendimento dos determinantes e dos fatores etiológicos do envelhecimento saudável - sejam eles relacionados às condições e desigualdades socioeconômicas, características individuais (biológicas, psicológicas, genéticas) e comportamentais, ambiente físico, acesso e utilização de serviços de saúde, ademais das diferenças culturais e de gênero - tornam-se fundamentais para subsidiar o planejamento de políticas de atenção à saúde da população idosa ${ }^{(4)}$.

O aumento da expectativa de vida traz novos contornos à população mundial. No que diz respeito ao Brasil, a Organização Mundial da Saúde estima que, até 2025, esse será o sexto país no mundo com maior número de idosos, alcançando 30 milhões de pessoas. Quanto à população feminina, a estatística mostra que é mais numerosa que a masculina. No último censo, realizado, pelo Instituto Brasileiro de Geografia e Estatística, foram contabilizadas 8.549.259 pessoas idosas do sexo masculino e 10.732 .790 do sexo feminino ${ }^{(5)}$.

Envelhecimento populacional é uma realidade em todo mundo e, chama a atenção que dessa população idosa brasileira, que já viveu 100 anos ou mais, quase 90\% são mulheres. Essa situação imprime uma importante característica às populações envelhecidas que é a sua feminização ${ }^{(6)}$. 
As mudanças sociais estão influenciando os modos de envelhecer da mulher, pois envelhecer é determinado não só pela cronologia e por fatores físicos, mas também pela condição social em que vivemos e pela singularidade individual de cada mulher idosa na sociedade ${ }^{(7)}$.

O prolongamento da existência só pode ser considerado uma conquista humana na medida em que agrega qualidade aos anos adicionais de vida, inclusive para indivíduos que tenham algum grau de fragilidade ou incapacidade, ou ainda, que necessitem de algum tipo de cuidado. Conforme destaca o marco político 'Envelhecimento Ativo', ações voltadas à população idosa devem oportunizar a saúde, a segurança pessoal e a participação contínua do idoso nas questões sociais, econômicas, culturais e civis de modo geral (4).

Nesse processo de envelhecimento a mulher passa pelo climatério, um período transicional, polêmico e crítico. Fase biológica da mulher, compreendendo a transição entre o período reprodutivo e o não reprodutivo, com início por volta dos 35 anos e encerrando-se aos 65 anos. Abrange a menopausa, correspondendo ao último ciclo menstrual, reconhecido depois de passados 12 meses da sua ocorrência, o que, em geral, se dá por volta dos 48 aos 50 anos de idade ${ }^{(6)}$.

Portanto, o envelhecimento da população é algo crescente mundialmente e deve se salientar que o processo de envelhecimento saudável e ativo é algo resultante de um conjunto de fatores que promovem uma melhor qualidade de vida para os idosos.

A partir da problemática da pesquisa o objetivo do presente estudo é analisar e descrever a prática e o conhecimento da mulher idosa sobre como manter um envelhecimento ativo e saudável e as dificuldades encontradas.

\section{MÉTODOS}

Trata-se de uma pesquisa de campo descritiva, com abordagem qualitativa. $\mathrm{O}$ cenário da pesquisa foi em um Centro de Convivência, de caráter público, situado na cidade de Teresina, Piauí, Brasil, onde são desenvolvidas atividades com: artesanato, canto e coral, educação física e orientação sexual sócio pedagógica. Participaram do estudo 12 mulheres idosas que frequentem o Centro de Convivência. Os critérios de inclusão foram idosas que frequentem o Centro de Convivência há pelo menos seis meses, com idade a partir de 60 anos e que concordem em participar da pesquisa por meio da assinatura do Termo de Consentimento Livre e Esclarecido (TCLE). Excluiu-se idosas que tenham um resultado do Mini Exame do Estado Mental (MEEM) inferior a uma pontuação de 27 pontos. Esse valor classifica como uma idosa com baixa cognição, estando assim não apta para 
responder as perguntas e que apresentem discordância na assinatura do TCLE.

Para identificar a cognição das idosas foi realizado o MEEM que é constituído de duas partes, uma que abrange orientação, memória e atenção, com pontuação máxima de 21 pontos e, outra que aborda habilidades específicas como nomear e compreender, com pontuação máxima de 9 pontos, totalizando um escore de 30 pontos ${ }^{(8)}$. As idosas foram nomeadas com nome "idosa" seguida de numeração arábica de ordem crescente, como forma de preservar a identidade das mesmas, em cumprimento as exigências da Resolução 466/12 do CNS ${ }^{(9)}$. A coleta de dados foi realizada após aprovação pelo Comitê e Pesquisa das instituições coparticipantes. Após o esclarecimento sobre os objetivos e metodologia da pesquisa, com consequente assinatura do TCLE, os dados da pesquisa foram coletados em salas reservadas, garantindo a intimidade e dignidade do entrevistado, e evitando também interferências por parte de terceiros. Estas foram gravadas, após o consentimento dos participantes e transcritas na íntegra, preservando a fala dos mesmos.

O instrumento para produção de dados foi um roteiro de entrevista semiestruturado detalhado e organizado, com perguntas abertas e fechadas, em que os entrevistados têm a possibilidade de discorrer sobre o tema proposto. Ressalta-se que a data da entrevista foi previamente agendada, respeitando a disponibilidade de tempo dos participantes e visando não inferir nas suas atividades. $\mathrm{O}$ tempo de duração da participação dos participantes nesta pesquisa teve duração em torno de trinta minutos.

A análise dos dados se deu por meio da técnica de análise de conteúdo, cuja finalidade é analisar a partir das falas dos entrevistados, organizá-las em categorias analíticas que atendam aos objetivos da pesquisa, síntese e interpretação dos resultados ${ }^{(10)}$. Primeiramente, as falas foram transcritas na íntegra, em seguida realizadas leituras e releituras flutuantes, com a finalidade de tomar contato exaustivo com as informações colhidas. Posteriormente, se processou a organização dos dados transcritos de cada entrevista, destacando os aspectos relevantes e comparando-os com a literatura. Estabeleceu-se classes analíticas a partir dos objetivos e do roteiro de entrevista.

A análise foi executada após a reprodução das gravações das falas das entrevistadas na íntegra, e em seguida, rigorosamente lidas e comparadas ao roteiro, para garantir qualidade do conteúdo. Após esta etapa realizou-se a análise de conteúdo de Minayo ${ }^{(10)}$.

Inicialmente buscou-se fazer uma leitura do conjunto do material selecionado, de forma completa. A partir dessa leitura 
analisou-se a busca de uma visão de conjunto. Foi compreendida as particularidades do conjunto do material analisado e elaborados pressupostos iniciais que serviram de baliza para a análise e a interpretação do material, logo após serão escolheu-se formas de classificação inicial, como também determinou-se os conceitos teóricos que orientaram a análise ${ }^{(10)}$.

$\mathrm{Na}$ segunda etapa, efetuou-se exploração do material mostrando a análise propriamente dita. A distribuição de trechos, depois foi feita leitura dialogando com as partes dos textos da análise ordenando cada assunto, para análise dos diferentes núcleos de sentido presentes na várias classes do esquema de classificação, afim de obter temáticas mais amplas ou eixos em torno dos quais pode-se discutir as diferentes partes dos textos analisados, em seguida reagrupou-se as partes dos textos por temas encontrados, para redação por tema, de modo que der conta dos sentidos dos textos e de sua articulação com conceitos teóricos que orientam a análise. No final foi implementada uma síntese interpretativa por meio de uma redação que possa falar de temas com objetivos, questões e pressupostos da pesquisa (10).

\section{RESULTADOS E DISCUSSÃO}

A seguir, as categorias temáticas que surgiram do estudo, de acordo com a similaridade semântica, que representam as descrições das falas das idosas entrevistadas.

\section{Práticas das idosas para um envelhecimento saudável e ativo}

“[...] Eu gosto muito de ler, fazer palavras cruzadas, atividade física, participo das atividades, não fico só em casa, eu saio para o shopping por exemplo." (1)

"[...] Eu me cuido, tomo os remédios direito, faço exercícios, vou ao médico de 3 em 3 meses." (3)

"[...] Faço academia, me movimento e faço as coisas em casa, no final de semana gosto de beber com as minhas amigas." (4)

“[...] Prático ginástica, não sou de ficar doente e sempre vou ao médico." (7)

"[...] Eu faço tudo, eu me cuido, lavo e passo roupa, passeio, sou ativa no que eu posso." (6)

"[...] Atualmente faço trabalhos manuais, faço flores, tapetes, costuro." (12)

As opiniões de idosos sobre as atividades que poderiam contribuir para melhorar a sua qualidade de vida, evidenciou que as atividades propostas pelos idosos são as atividades de lazer como ir ao cinema, praticar esportes, jogos e brincadeiras, 98,5\%. Pescaria e Boliche com 98\% das respostas e aulas de canto/música, 97,5\%. Os passeios e as aulas de dança também são importantes e foram citados em $96,5 \%$ e $94 \%$ das respostas 
respectivamente. Entre as menos quistas estão as atividades manuais com $87 \%$ das opiniões em favor de sua realização ${ }^{(11)}$.

A atividade física regular melhora a força, a massa muscular e a flexibilidade articular. Também atua como método de prevenção de doenças neurológicas, como esclerose múltipla e doença de Alzheimer ${ }^{(12)}$.

A população idosa deve ser considerada como sujeito ativo na promoção de sua saúde e na manutenção de sua autonomia, em que sejam realizadas orientações sobre os cuidados e o apoio pela família e pelos serviços de saúde na execução das atividades físicas que não necessitem de auxílio ou supervisão, mas que permitam desenvolver as atividades físicas sem risco (13)

Além da atividade física ser um fator importante na qualidade de vida das pessoas, há evidências de que a população que mais se beneficia com esta prática é a população de idosos. Neste sentido, a atividade física na velhice mostra-se promissora em despertar no idoso a manutenção a saúde e interação com o meio ${ }^{(12)}$.

\footnotetext{
"[...] Envelhecer ativamente é envelhecer com saúde, através de caminhada, passeios, diversão com a família." (5)

“[...] Faço tudo, não espero por ninguém, eu brinco com os meus netos, gosto de sair para beber de vez enquanto." (8)
}

"[...] Eu nunca me importei em envelhecer, eu sabia que um dia iria ficar mais velha, mais para mim envelhecer com saúde, é viver feliz, passear com a família." (9)

"[...] Ando muito nas casas das minhas irmãs para conversar, me distrair." (11)

Em uma pesquisa realizada com 246 idosos constatou-se que quanto à prática do etilismo foi referida por apenas $8,9 \%$ dos participantes, contudo $11,0 \%$ já fizeram uso do álcool em algum momento de sua vida. Portanto, apesar de a maioria dos idosos não usar álcool, é necessária atenção a este fator de risco, visto que se sabe que o alcoolismo é um significativo problema de saúde pública no país, levando a inúmeras patologias ${ }^{(14)}$.

A “união" familiar surgiu como uma categoria neste estudo quando os idosos passaram a associar o desempenho adequado na função de cuidado, tanto a avós como a netos, à manutenção de laços entre os membros da família. Grande parte dos avós pode desfrutar mais da companhia dos netos, por ter um tempo livre maior, o que contribuiria para fortalecer os vínculos dentro da família ${ }^{(15)}$.

\section{"[...] Faço ginástica, educação} física, jogo da memória e tenho uma boa alimentação." (1)

"[...] Me alimento bem, só como mais comidas saudáveis, frutas e verduras que eu acho essencial para um bom envelhecimento." (10) 
Em um estudo feito com 1705 idosos sobre as variáveis estudadas observou-se que a maioria manteve-se com companheiro $(53,4 \%)$, permaneceu morando acompanhado (76,0\%), manteve-se sem trabalhar $(78,0 \%)$, com percepção positiva de saúde $(45,0 \%)$, sem fumar $(57,5 \%)$, sem consumir bebida alcoólica $(53,3 \%)$, inativo/insuficientemente ativo $(56,3 \%)$, com consumo $<5$ porções diárias de frutas e hortaliças $(57,3 \%)$, com excesso de peso $(47,0 \%)$, sem dependência $(60,8 \%)$, com rastreamento negativo para deficit cognitivo $(69,4 \%)$, sem sintomas depressivos $(72,0 \%)$ e sem quedas no último ano $(60,6 \%)^{(4)}$.

A ginástica aeróbica como primeira opção de modalidade de atividade física na velhice, seguida da hidroginástica, da caminhada e por último a musculação que auxiliam na qualidade de vida dos idosos ${ }^{(16,}$ 17).

\section{Dificuldades das idosas para o envelhecimento ativo}

“[...] Eu estou nessa idade e só tomo remédio para hipertensão, mas, todos os anos faço exames." (1)

"[...] Tenho diabetes, mais sempre tento manter o controle." (12)

Em estudo feito com 1.593 indivíduos com 60 anos ou mais, o percentual de indivíduos que referiram ter hipertensão, problema cardíaco e diabetes foram, respectivamente, de 55,3;29,6 e 15,1\%. A prevalência de depressão na população idosa da área urbana de Bagé foi de 18,0\%, sendo 20,5\% nas áreas cobertas pela ESF e 15,1\% nas áreas da atenção básica tradicional ${ }^{(18)}$.

Em outro estudo realizado com 100 idosos apontou que entre as doenças referidas pelos idosos, prevalecem a hipertensão arterial sistêmica em $60 \%$ e as cardiopatias, em $22 \%$. Em relação à funcionalidade familiar, os resultados apontam que $80 \%$ dos idosos possuem elevada disfuncionalidade ${ }^{(18)}$.

Quanto à caracterização clínica de 52 idosos, as comorbidades mais frequentes foram: hipertensão arterial, $25(48,1 \%)$; osteoporose, 18 (34,2\%); diabetes, 10 $(19,2 \%)$; gastrite, $08(15,4 \%)$ e incontinência urinária, $08(15,3 \%)^{(19)}$.

"[...] As vezes quando acordo sinto dores no corpo, principalmente no joelho." (2)

"[...] No momento a dificuldade que eu sinto é em fazer as tarefas de casa, pois sinto muitas dores na coluna e no joelho." (5)

“[...] Depois que operei da minha perna, eu ando bem devagar $e$ isso me atrapalha no meu dia a dia, não consigo subir em nada mais." (6)

“[...] Não pego muito peso, pois sinto muitas dores na coluna." (8)

“[...] Sinto dores no corpo, mais quando faço atividade física melhora." (10)

No mesmo estudo realizado em Bagé com 1.593 indivíduos com 60 anos ou mais constatou-se que a prevalência de 
incapacidade para atividades básicas da vida diária foi de $10,6 \%$ e a prevalência para incapacidade para as atividades instrumentais da vida diária foi de $34,2 \%{ }^{(18)}$.

Em estudo feito com 52 idosos, as principais demandas relacionadas à necessidade de mover-se e manter uma postura adequada identificadas nos idosos foram: dificuldades para mover-se, 22 $(42,3 \%)$; rigidez articular, $31(59,6 \%)$; dor ao mover-se, 30 (57,7\%); não praticar atividade física, 37 (71,1\%); risco para quedas, 35 $(67,3 \%)$. Apesar desses problemas, apenas 03 $(5,8 \%)$ faziam uso de auxílio locomoção bengala, e $09(17,3 \%)$ idosos reconheciam a necessidade de ajuda para mover-se e manter uma postura adequada ${ }^{(19)}$.

"[...] Me sinto sozinha, porque moro só com o meu marido e os meus filhos pouco me visitam." (3)

"[...] Problemas familiares que me preocupam e que influenciam na minha saúde física e mental."

As percepções pessoais dos membros de uma família acerca de certas variáveis, como qualidade de vida e estilo de vida, podem influir na dinâmica familiar, fazendo-a mais ou menos adaptativa, mais ou menos funcional, em face da situação que se apresenta como fato novo a enfrentar: um membro idoso que adoece e se torna cada dia mais frágil e dependente, exigindo cuidados e criando impactos sobre as relações intrafamiliares ${ }^{(20)}$.
"[...] Eu me esqueço muito das coisas e isso está me incomodando." (7)

“[...] Eu ainda trabalho muito.” (9)

Em uma oficina de idosos feita em uma unidade básica de saúde, os motivos apresentados pelos idosos para participar das atividades foram estabelecidos em três categorias: medo de perder a memória, achar que já estava apresentando falhas na memória, vontade de participar de atividades de promoção da saúde. Sendo o primeiro e o terceiro os mais apontados ${ }^{(21)}$.

Para alguns dos idosos em estudo, a velhice está associada à decadência, à dependência e à incapacidade para as atividades simples da vida diária, como, por exemplo, andar, vestir-se, cuidar da casa e dos netos e também além dos aspectos biológicos relacionados ao envelhecimento, os aspectos psíquicos que envolvem a capacidade de raciocínio, independentemente de outros fatores, foram relatados como determinantes do envelhecimento ${ }^{(22)}$.

Conhecer a percepção dos idosos sobre o processo de envelhecimento é útil para que se direcionem propostas de atividades sociais a essa clientela, já que há influências culturais em relação à forma como a saúde e o processo de degeneração senil são percebidos, pois é a partir de uma análise comparativa com demais indivíduos que se delineia a condição de saúde e de velhice ${ }^{(22)}$. 


\section{CONCLUSÃO}

Conclui-se que o envelhecimento ativo é encarado como um "processo de otimização das oportunidades de saúde, participação e segurança, com o objetivo de melhorar a qualidade de vida à medida que as pessoas ficam mais velhas garantindo assim uma melhor qualidade de vida.

Nesta pesquisa observou-se as boas práticas para um envelhecimento saudável e ativo, como também as dificuldades encontradas, no qual foi constatado que as idosas tinham principalmente uma boa alimentação, realizavam atividade física, atividades de lazer que contribuam para um bom envelhecimento. E como principais dificuldades para manter um envelhecimento ativo, nota-se o aparecimento de doenças crônicas, como hipertensão e diabetes mellitus, dores na coluna, sentimento de solidão.

É notório dizer que o lazer e a qualidade de vida estão intimamente ligados aos aspectos das condições humanas, podemos definir que a trajetória de vida de um indivíduo, quando bem desenvolvida dentro de parâmetros saudáveis a serem seguidos, leva a uma longevidade atuante na sociedade.

Portanto, conhecer as diferentes dimensões que envolvem a concepção de velhice pode incentivar o profissional de saúde a adotar evidências que guardam relação com o cuidado no processo saúde/doença, resultando na melhoria e na qualificação da assistência ao idoso.

\section{REFERÊNCIAS}

1. Costa FODB, Leitão FJP, Massart A. Avaliação da Aptidão Física de um Grupo de Idosos entre os 75 e 95 Anos da Zona de Sertã [Monografia]. Universidade de Coimbra; 2008 [acesso em 4 mai 2018]. Disponível em: https://estudogeral.sib.uc.pt/handle/10316/106 29

2. Organização Mundial De Saúde (OMS). Envelhecimento Ativo: Uma política de Saúde: [em linha]. [Internet]. 2005 [acesso em 4 mai 2018]. Disponível em: http://bvsms.saude.gov.br/bvs/publicacoes/en velhecimento_ativo.pdf

3. Mantovani EP, Lucca SR, Neri, AL. Associações entre significados de velhice e bem-estar subjetivo indicado por satisfação em idosos. Rev. bras. geriatr. gerontol. [Internet]. 2016 [acesso em 8 mai 2018];19(2). Disponível em: http://www.scielo.br/scielo.php?pid=S1809$98232016000200203 \&$ script $=$ sci_abstract $\&$ tl $\mathrm{ng}=\mathrm{pt}$

4. Confortin SC, Schneider IJC, Antes DL, Cembranel F, Ono LM, Marques LP et al . Condições de vida e saúde de idosos: resultados do estudo de coorte EpiFloripa Idoso. Epidemiol. Serv. Saúde [Internet]. 2017 [acesso em 20 mai 2018]; 26(2): 305$317 . \quad$ Disponível em: http://www.scielo.br/scielo.php?script=sci_art text\&pid=S2237-

$96222017000200305 \& \operatorname{lng}=\mathrm{en}$.

5. Caldeira S, Merighi MAB, Muñoz LA, Jesus MCP, Domingos SRF, Oliveira DM. O enfermeiro e o cuidado à mulher idosa: abordagem da fenomenologia social. Rev. Latino-Am. Enfermagem [Internet]. 2012 [acesso em 20 mai 2018]; 20(5): 888$895 . \quad$ Disponível em: http://www.scielo.br/scielo.php?script=sci_art 
text\&pid=S0104-

$11692012000500010 \& \operatorname{lng}=\mathrm{en}$.

6. Silva GF, Moura MAV, Almeida MV de S, Sá SPC, Queiroz ABA. Influências do climatério para o envelhecimento na percepção de mulheres idosas: subsídios para a enfermagem. Rev. Eletr. Enf. [Internet].2016 [acesso em 01 jun de 2018]; 17(3). Disponível em: https://revistas.ufg.br/fen/article/view/29072.

7. Lima LCVD, Bueno CMLB. Envelhecimento e gênero: a vulnerabilidade de idosas no brasil. Revista Saúde e Pesquisa. [Internet]. 2009 [acesso em 01 jun de 2018]; 2 (2). Disponível em: https://periodicos.unicesumar.edu.br/index.ph p/saudpesq/article/view/1173.

8. Folstein MF, Folstein SE, Mchugh PR. Mini-menta state". A practical method for grading the cognitive state of patients for the clinician. J Psychiatr Res. [Internet]. 1975 [acesso em 03 jun de 2018]; 12 (3). Disponível em: https://www.sciencedirect.com/science/article /abs/pii/0022395675900266?via\%3Dihub.

9. Ministério da Saúde (BR). Diretrizes e normas regulamentadoras da pesquisa envolvendo seres humanos. Brasil [Internet]. 2012 [acesso em 05 jul de 2018]. Disponível em:

https://bvsms.saude.gov.br/bvs/saudelegis/cns /2013/res0466_12_12_2012.html.

10. Minayo MCS. O desafio do conhecimento: Pesquisa Qualitativa em Saúde. 12 ${ }^{\mathrm{a}}$ ed. São Paulo: Hucitec-Abrasco, 2010.

11. Almeida MAG, Wagner V, Oliveira LP. Lazer nos centros de convivência dos municípios da região norte do Paraná. R. bras. Ci.e Mov 2018 [acesso em 05 jul de 2018]; 26(3):156-164. Disponível em: https://pesquisa.bvsalud.org/portal/resource/pt /biblio-967129.

12. Rocha ECA. Prática de Atividades Físicas $\mathrm{X}$ Saúde do Idoso. Conteúdo Jurídico, Brasília-DF, 2012.
13. Machado FN. Capacidade e desempenho para as atividades básicas de vida diária: um estudo com idosos dependentes. [dissertação] Universidade Federal de Minas Gerais; 2010. [acesso em 10 set 2018]. Disponível em: http://www.enf.ufmg.br/pos/defesas/485M.P DF.

14. Ferreira JDF, Moreira RP, Maurício TF, Lima PA, Cavalcante TF, Costa EC. Fatores de Risco para Doenças Cardiovasculares em Idosos. Rev enferm UFPE on line [internet] 2017 [acesso em 05 jul de 2018]; 11 (12): 4895-905.

Disponível em: https://doi.org/10.5205/1981-8963v11i12a15182p4895-4905-2017.

15. Wegner E, Brittes-Benitez L. O idoso no contexto familiar: a função de cuidado. Revista Jovens Pesquisadores [Internet] 2013; [acesso em 07 jul de 2018] 3(2). Disponível em: https://online.unisc.br/seer/index.php/jov enspesquisadores/article/view/4089.

16. Santana MS. Significado da atividade física para Práticas de saúde na terceira idade. Estudos Interdisciplinares sobre o Envelhecimento [internet] 2010 [acesso em 15 out 2018]; 15(2) Disponível em: http://seer.ufrgs.br/index.php/Ver Envelhecer/article/view/11995/11479.

17. Queiroz Junior CA et al. Motivos de adesão das mulheres idosas participantes dos programas Públicos de exercícios físicos em Uberaba-Mg.2012. Coleção Pesquisa em Educação Física [internet] 2012 [acesso em 15 out 2018]; 11(4). Disponível em: https://fontouraeditora.com.br/periodico/uploa d/artigo/967_1503345505.pdf.

18. Bretanha AF, Facchini LA, Nunes BP, Munhoz TN, Tomasi E, Thumé E. Sintomas depressivos em idosos residentes em áreas de abrangência das Unidades Básicas de Saúde da zona urbana de Bagé, RS. Rev. bras. epidemiol. [Internet] 2015 [acesso em 15 out 2018]; 18(1): 1-12. Disponível em: http://www.scielo.br/scielo.php?script=sci_art text\&pid=S1415790X2015000100001\&lng=en. 
19. Clares JWB, Freitas MC, Borges CL. Fatores sociais e clínicos que causam limitação da mobilidade de idosos. Acta paul. enferm. [Internet]. 2014 [acesso em 15 out 2018]; 27(3): 237-242. Disponível em: http://www.scielo.br/scielo.php?script=sci_art text\&pid=S0103-

$21002014000300237 \& \operatorname{lng}=$ en.

20. Gonçalves LTH, Leite MT, Hildebrandt LM, Bisogno SC, Biasuz S, Falcade BL. Convívio e cuidado familiar na quarta idade: qualidade de vida de idosos e seus cuidadores. Rev. bras. geriatr. gerontol. [Internet]. 2013 [acesso em 20 out 2018]; 16(2): 315$325 . \quad$ Disponível em: http://www.scielo.br/scielo.php?script=sci_art
text\&pid=S1809$98232013000200011 \& \operatorname{lng}=\mathrm{en}$.

21. Wanderbroocke AC, Folly PP; Maba PC et al. Oficina de memória para idosos em uma unidade básica de saúde: um relato de experiência. Psic. Rev, 2015.

22. Faller JW, Teston EF, Marcon SS. A velhice na percepção de idosos de diferentes nacionalidades. Texto contexto - enferm. [Internet]. 2015 [acesso em 20 out 2018]; 24(1): 128-137. Disponível em: http://www.scielo.br/scielo.php?script=sci_art text\&pid=S0104-

07072015000100128\&lng=en.

Submissão: 2021-04-28

Aprovado: 2021-04-28 